\title{
Ecologia Urbana: histórico, definições e abordagens interdisciplinares
}

\author{
José Lucas dos Santos Oliveira $₫$ ๑, Joel Silva dos Santos \\ Programa de Pós-Graduação em Desenvolvimento e Meio Ambiente, Universidade Federal da Paraíba, \\ João Pessoa, 58051-900, Paraíba, Brasil. *lucasoliveira.ufcg@gmail.com
}

Received: 17 julho 2021 / Accepted: 20 setembro 2021 / Published online: 30 setembro 2021

\begin{abstract}
Resumo
O objetivo deste artigo foi realizar um resgate histórico do surgimento, definições, e abordagens da Ecologia Urbana. Para a realização da pesquisa, utilizou-se principalmente artigos publicados em periódicos acadêmicos nacionais e internacionais. A Ecologia Urbana foi criada em 1920 pela Escola de Chicago, emergindo da necessidade de se compreender as dinâmicas de funcionamento das cidades, abordando alterações antrópicas em áreas urbanas e sua interação com os ecossistemas naturais e o ambiente construído. As pesquisas em Ecologia Urbana podem ser estritamente biológicas (Ecologia na Cidade), ou possuir enfoque interdisciplinar com a interação das ciências naturais e sociais (Ecologia da Cidade). A abordagem interdisciplinar da Ecologia Urbana é imprescindível para a promoção da sustentabilidade ecológica e socioeconômica em áreas urbanas, servindo de base para o estabelecimento de estratégias e Políticas Públicas de planejamento e gestão sustentável das cidades.
\end{abstract}

Palavras-chave: Cidade sustentáveis, escola de Chicago, interdisciplinaridade.

\section{Urban Ecology: history, attributions and methodological approaches interdisciplinary}

\begin{abstract}
The objective of this article was to carry out a historical review of the emergence, definitions, and approaches of Urban Ecology. To carry out the research, mainly articles published in national and international academic journals were used. Urban Ecology was created in 1920 by the Chicago School, emerging from the need to understand the dynamics of how cities function, addressing human changes in urban areas and their interaction with natural ecosystems and the built environment. Research in Urban Ecology can be strictly biological (Ecology in the City), or have an interdisciplinary approach with the interaction of natural and social sciences (Ecology of the City). The Urban Ecology approach interdisciplinary is essential for the promotion of ecological and socio-economic sustainability in urban areas, serving as a basis for the establishment of strategies and Public Policies for planning and sustainable management of cities.
\end{abstract}

Keywords: Sustainable cities, school of Chicago, interdisciplinarity.

\section{Introdução}

O surgimento das cidades ocorreu a partir da necessidade da espécie humana em se agrupar e unir-se em busca de proteção, de água e alimentos para sobreviver. O padrão de vida baseado em aglomerações humanas fixadas em determinados ambientes ocasionou mudanças nas necessidades básicas do ser humano até então desconhecidas, como por exemplo, criação das cidades e estratificação estrutural da sociedade (Adler e Tanner, 2015). Tais alterações no modo de vida e nos padrões de consumo humano, exerceram forte pressão nos sistemas ambientais e nas diversas paisagens naturais.

Historicamente a sociedade moderna (re)produz constantemente o espaço geográfico, com a transformação das paisagens naturais em ecossistemas urbanos. Nesse contexto, as cidades têm seguido a lógica capitalista de produção com a transformação do espaço geográfico por ações antrópicas. Isso tem gerado uma série de impactos socioambientais adversos, com fortes desigualdades socioespaciais e o comprometimento da qualidade de vida das populações que ocupam esses espaços artificializados.

Seguindo esta lógica, convém destacar que o aumento da população global e a busca por melhores condições de vida têm resultado no crescimento acelerado das cidades, desencadeando degradações ambientais e desigualdades socioespaciais materializadas no espaço geográfico. Nessa perspectiva, Rodrigues e Victor (2014) caracterizam as cidades entre os principais fenômenos sociais que ocorreram na história da humanidade com forte poder de impacto nos sistemas socioambientais.

Tais mudanças contribuem para a domesticação de ecossistemas e paisagens terrestres no ambiente construído (Wu, 2014) e o afloramento de novas pandemias como o coronavírus, que causa a COVID-19, doença zoonótica 
emergente que está sendo associada a modificação de paisagens naturais por atividades antrópicas (Pnuma, 2020).

Com mais da metade da população mundial residindo atualmente em áreas urbanas e gerando impactos nos sistemas socioambientais, o estudo da Ecologia Urbana se reveste de forma significativa para a compreensão do meio ambiente e dos serviços ecossistêmicos em interação com os sistemas socioeconômicos. Nesse sentido, faz-se necessário destacar a importância da Ecologia Urbana para a promoção de cidades sustentáveis, visto que se caracteriza como uma disciplina holística e multidisciplinar, que integra o conhecimento das ciências naturais e sociais (Niemelä, 2014).

A atuação da Ecologia Urbana nos ecossistemas urbanos é complexa, devido as cidades estarem em constante processo de mudança e desequilíbrio, portanto, é preciso a integração entre o social, ambiental e a infraestrutura para a construção de uma cidade mais sustentável, igualitária e resiliente (McPhearson et al., 2016).

Diante deste contexto, o objetivo deste trabalho foi realizar um resgate histórico do surgimento, definições, e abordagens metodológicas da Ecologia Urbana.

\section{Contextualização histórica e expansão da Ecologia Urbana antes e pós século XXI}

O termo Ecologia surgiu em 1866, derivado das palavras gregas "oikos" e "logos", que juntos significam a "ciência do habitat" e foi citado pela primeira vez pelo zoólogo alemão Ernst Haeckel (Haeckel, 1866). Nas primeiras observações de Haeckel, as espécies foram consideradas interdependentes de seus respectivos habitats e a Ecologia passou a ser definida como ciência e também uma disciplina científica (Nucci, 2007).

Adler e Tanner $(2015$, p. 3) ressaltam que a Ecologia, de forma geral, se desenvolveu enquanto ciência no final do século XIX devido à elevação do conhecimento integrado da "História Natural detalhada das espécies e dos seus hábitos, a ênfase de Darwin na interação entre espécies e na mudança destas no decorrer do tempo e o melhor entendimento da fisiologia de plantas e animais".

Dessa forma, a Ecologia passou a ser conceituada dentro do escopo das Ciências Naturais, como as interações das espécies com o ambiente, ou seja, a ciência das relações (Lawrence, 2003). Steiner (2008) complementa que a Ecologia representa o fluxo contínuo de relação e reciprocidade dos organismos com o ambiente físico e biológico.

Na concepção de Bartot el al. (2019), a Ecologia é uma ciência que estuda as interrelações entre diferentes organismos/espécies e destes com o meio ambiente, independente de escalas temporais ou espaciais que estejam localizados.

Contudo, o estudo das complexas e diferentes interações do ser humano com o ambiente pode ser compreendido como Ecologia Humana e, atualmente, auxilia no entendimento dos processos que norteiam a vida humana no planeta Terra (Steiner, 2008; Silva e Bomfim, 2019). O termo Ecologia Humana foi criado por Robert Park e Ernest Burggess (Lawrence, 2003) entre as décadas de 1920 e 1930 (Nucci, 2007), e, na época, representava a organização espaço- temporal do ser humano e de suas relações com os processos do meio ambiente (Park; Burgess e Mckenzie, 1925).

A motivação de criação da Ecologia Humana partiu da necessidade de superar as limitações da Ecologia geral, que concentrou os objetivos de pesquisa sem integrar explicitamente os seres humanos enquanto espécie constituinte e interdependente da natureza (Silva e Bomfim, 2019). A Ecologia Humana necessitou então se desenvolver de forma interdisciplinar para compreender e refletir sobre as interrelações humanas com a natureza, que foram pouco exploradas pela Ecologia tradicional.

Destaca-se que antes do surgimento da Ecologia Humana, as Ciências Sociais contribuíram significativamente para os conhecimentos da área, pois buscava entender o funcionamento da realidade complexa vivenciada pelos Estados Unidos na época, considerando a transição de um país agrário para um grande centro industrial e econômico com destaque mundial, sendo acompanhado de um rápido crescimento populacional e demográfico que resultou em problemas socioambientais (Eufrasio, 2013) que posteriormente foram estudados pela Ecologia Humana.

O surgimento da Ecologia Humana foi um marco histórico, pois proporcionou que os estudos sobre organização social e distribuição espacial humana pudessem ser realizados (Lawrence, 2003). Relatos do século XIX e XX demonstram muitas interações entre as Ciências Sociais e Biológicas e, nesse contexto, a Ecologia Humana foi determinada como área integrante da geografia. Da mesma forma, os conhecimentos ecológicos também foram incorporados aos estudos sociológicos e antropológicos com a intenção de integrar os sistemas sociais com os sistemas naturais (Steiner, 2008).

Ainda diante deste cenário, Steiner (2008) também descreve que a forma de atuação da geografia no início do século XX estava ligada ao determinismo ambiental, por acreditar que o meio ambiente determinava todos os aspectos da vida humana, incluindo o comportamento. Nessa época, a Ecologia Humana, que também estava associada a geografia, acabou sendo sucumbida pelas críticas ao determinismo ambiental. Essa forma de pensamento era considerada limitada e o determinismo ambiental, segundo o autor, foi descreditado a partir de 1920.

É importante ressaltar que a criação da Ecologia Humana proporcionou importantes contribuições cientificas e sociais, ampliando o campo de atuação da Ecologia tradicional ao envolver e se concentrar no estudo das interações humanas com a natureza, e da natureza com o homem, visando reverter o panorama de degradação do planeta Terra ocasionado por ações antrópicas insustentáveis (Silva e Bomfim, 2019).

Sendo assim, nesse contexto de transições e mudanças paradigmáticas de pensamentos relacionados as interações ambientais e humanas, surge também, ainda na década de 1920, o conceito de Ecologia Urbana, por meio de discussões de um grupo de sociólogos da Universidade de Chicago. Nessa época, a Ecologia Urbana surgia como parte integrante e derivada dos conhecimentos da Ecologia Humana ( $\mathrm{Wu}$, 2014). 
A Escola de Chicago se caracterizava como um grupo seleto de pensadores sociólogos, que possuíam linhas de investigações ligadas ao Departamento de Sociologia de Chicago, cujo o interesse se voltava para os estudos sociológicos urbanos da cidade de Chicago. Ainda nesse período, os Estados Unidos elevavam o seu desenvolvimento socioeconômico, ampliando também o desenvolvimento intelectual com a construção de importantes universidades nos EUA, como a Universidade de Chicago, que concentrou os estudos sociológicos no país e elevou o reconhecimento da universidade ao aproximar-se da comunidade por meio de pesquisas direcionadas aos problemas sociais, estrutura urbana e ecológica, principalmente de Chicago (Eufrasio, 2013).

O grupo de sociólogos que constituíam a Escola de Chicago discutiam os conceitos da Ecologia numa perspectiva do ambiente urbano, que se destacou pelo trabalho desenvolvido na compreensão da vida e cultura urbana (Steiner, 2008), utilizando como base os conhecimentos ecológicos (Adler e Tanner, 2015), visando exemplificar como ocorriam as interações humanas nas cidades (Steiner, 2008).

A Escola de Chicago teve sua criação preconizada entre duas guerras mundiais no século XX, que consistia em um cenário delicado de constantes mudanças e conflitos no território americano (Eufrasio, 2013). Foi nesse panorama histórico que a Escola de Chicago foi fundada por Robert Park, Ernest Burgess e Roderick McKenzie (Osmond e Pelleri, 2017), onde esses e outros sociólogos estudaram a cidade de Chicago para demonstrar, usando o conceito de sucessão ecológica, como ocorriam os processos de ocupação urbana e crescimento da cidade desde o centro até as regiões periféricas, por meio de sucessões humanas (Steiner, 2008). Na Escola de Chicago, os termos de Ecologia Humana e Ecologia Urbana eram utilizados de forma semelhante para se referir as interações humanas e sua distribuição no ambiente (Lawrence, 2003).

A partir disso, com a ampliação dos conhecimentos, a Ecologia Urbana se expandiu pelos Estados Unidos por meio de importantes iniciativas de centros de pesquisa, resultando em conceituadas publicações que estimularam o desenvolvimento da área pelo país e, consequentemente, com estudos na Europa, China, África do Sul e outros países no mundo (McPhearson et al., 2016).

Os estudos de Ecologia Urbana desenvolvidos na Europa durante o século XIX visavam a compreensão da vida de organismos vegetais presentes em habitats urbanos e em áreas impactadas por ações antrópicas, sendo pioneiros na descoberta da diferença entre espécies nativas e introduzidas (Adler e Tanner, 2015).

Barot et al. (2019) retratam que, considerando a evolução histórica de pesquisas da área, os estudos de Ecologia Urbana têm se elevado em termos quantitativos, compreendendo cerca de 14\% de todos os estudos e publicações da Ecologia geral. De acordo com os autores, esses dados reportam o aumento da representatividade da Ecologia Urbana ao longo do tempo nas publicações mais recentes, principalmente quando comparados as primeiras pesquisas realizadas no ambiente urbano na década de 1990, no qual as publicações em periódicos de Ecologia somavam menos de 100 artigos publicados anualmente sobre a temática da Ecologia Urbana.
Na pesquisa de Barot et al., (2019), é retratado ainda que até os anos 2000 pouco se publicava sobre a Ecologia Urbana, pois a Ecologia ainda era compreendida como uma subdivisão da Biologia. Esse aumento exponencial nas pesquisas da área pode ser justificado por três fatores principais: criação de novos periódicos de Ecologia Urbana, motivando o desenvolvimento de pesquisas, o aumento da população urbana, que tem gerado impactos aos ecossistemas naturais dentro das cidades e o aumento dos cursos de graduação em Ecologia. Nesse sentido, vale destacar que os estudos de Ecologia Urbana são considerados relativamente recentes (Adler e Tanner, 2015).

No contexto atual, tem-se observado que a partir da contextualização histórica, mesmo com os avanços nas publicações e estudos de Ecologia Urbana no mundo, cerca de $90 \%$ das pesquisas da área foram realizadas nos Estados Unidos e Europa, por isso, ainda se observa em alguns países, como por exemplo o Brasil, as pesquisas de Ecologia Urbana se apresentarem de forma incipientes (Rumble; Angeoletto; Connop; Goddard e Nash, 2019).

Mesmo diante da pouca representatividade da Ecologia Urbana em alguns países, a expansão dos conhecimentos e pesquisas da área criou conceitos diversos que foram utilizados por diferentes pesquisadores, como os ecólogos, geógrafos, pesquisadores de planejamento urbano e ciências sociais (Wu, 2014). Ou seja, a Ecologia Urbana foi sendo moldada em virtude do viés naturalista ou social impresso pelo pesquisador e, também, por sua base conceitual interdisciplinar.

Sendo assim, o surgimento da Ecologia Urbana proporcionou a construção de debates sobre a sustentabilidade e resiliência das cidades em um contexto multidisciplinar (McPhearson et al., 2016). Desde então, os estudos de Ecologia Urbana tornaram-se imprescindíveis para a compreensão das cidades enquanto ecossistemas, e para a conservação ambiental dentro dos espaços urbanos, pois a visão da cidade como ecossistema integra as dimensões sociais, econômicas e ecológicas.

\section{Características gerais e conceitos fundamentais que embasam os estudos de Ecologia Urbana}

Como visto anteriormente, a Ecologia Urbana surge em um contexto onde a cidade tem se tornado o principal habitat humano no planeta, e onde frequentemente se observa a dominância e modificação dos ecossistemas naturais pelas ações antrópicas (Osmond e Pelleri, 2017). Todos esses impactos socioeconômicos e socioambientais não são totalmente conhecidos (Wu, 2014). Daí a importância da Ecologia Urbana para a compreensão de tais processos. Contudo, sabe-se que os impactos ao meio ambiente podem adquirir proporções locais, regionais e globais e não são tão simples de serem compreendidos (Wu, 2008).

Nessa perspectiva, a Ecologia Urbana apresenta muitas abordagens e conceitos publicados em artigos científicos das mais diversas áreas do conhecimento que são adaptados para realidades diferentes e, por isso, nem sempre podem ser comparados (McPhearson et al., 2016). Essa amplitude de conceitos é resultado das diversas fontes e áreas de 
conhecimento interdisciplinares que originaram a Ecologia Urbana (Adler e Tanner, 2015) e, também, das recentes pesquisas desenvolvidas envolvendo a temática (Barot et al., 2019).

Considerando a complexidade do tema, um conceito universalmente proposto para a definição de Ecologia Urbana ainda é amplamente discutido (Niemelä, 1999; Wu, 2014). Por isso, para uma melhor compreensão do que vem a ser Ecologia Urbana, é necessário conhecer além do conceito de Ecologia, o conceito de urbano.

No que diz respeito ao conceito do que venha a ser urbano, Wu (2014) destaca a necessidade de três características básicas essenciais, que variam de acordo com a dimensão e a localização da área: tamanho total, densidade populacional e superfícies impermeáveis ou estruturas construídas. A densidade populacional e as áreas construídas também são citadas por Niemelä (1999) para caracterizar uma área urbana.

O ambiente urbano compreende também áreas além dos domínios da cidade, mas que se interconectam de alguma forma com esta, por participarem ativamente da absorção de resíduos produzidos, como também pelo fornecimento de energia e materiais (Angeoletto et al., 2015). Os espaços urbanos formam uma paisagem que se caracteriza por conectar diferentes construções humanas (residenciais, comerciais e industriais) por meio de estradas e rodovias, possuindo, em alguns casos, a presença de cobertura vegetal entre essas áreas (Tonyaloglu, 2020).

Nesse contexto, Wu (2014, p. 213) define a Ecologia Urbana como "o estudo de padrões espaço-temporais, impactos ambientais e sustentabilidade da urbanização com ênfase na biodiversidade, processos ecossistêmicos e serviços do ecossistema". O conceito apresentado por Wu (2014) aborda as diversas dimensões da Ecologia Urbana e suas perspectivas atuais.

A Ecologia Urbana seria então uma área e/ou subárea dentro da Ecologia, que possui como fundamentos e princípios os conhecimentos, teorias e métodos originados das ciências naturais e sociais visando a compreensão e o estudo de padrões e processos que determinam os ecossistemas urbanos (Grimm et al., 2008), revolucionando o pensamento humano de maneira sistemática acerca das questões que envolvem o urbano e suas relações com o meio ambiente (Batitucci; Cortines; Almeida e Almeida, 2019).

Nesse sentido, Batitucci et al. (2019, p. 4) destacam ainda que a Ecologia Urbana busca estudar "o ambiente urbano e todas as complexas interações que ocorrem nele, investigando as relações dos ocupantes das áreas urbanas, sejam pessoas, animais, indústrias ou outras coisas semelhantes, com seus impactos sobre o meio ambiente". Esse conceito de Ecologia Urbana se apresenta de forma mais complexa, relacionando a necessidade de compreender as interações das áreas construídas com o ambiente natural.

Outros conceitos recentes de Ecologia Urbana encontrados na literatura são descritos por Pickett (2020), que defende a ideia de Ecologia na Cidade e Ecologia da Cidade; Leveau (2020) destaca a interdisciplinaridade existente na Ecologia Urbana e sua atuação nos estudos da coexistência das espécies biológicas com o ambiente urbano, na qual, esta última ideia, também é defendida por Fellowes (2019).

Dessa forma, a Ecologia Urbana por essência perpassa uma abordagem multidisciplinar, direcionando suas diversas abordagens e perspectivas também para uma visão transdisciplinar, que deve envolver organizações governamentais para auxiliar na tomada de decisões que contemplem os mais impactados dentro das cidades (McPhearson et al., 2016). É importante destacar que a Ecologia Urbana é considerada uma ciência aplicada (Niemelä, 1999), dessa forma, seus conhecimentos são direcionados para a resolução de problemáticas que afetam a dinâmica das cidades e de seus ecossistemas.

$\mathrm{Wu}$ (2014, p. 218), afirma que as pesquisas desenvolvidas na área de Ecologia Urbana são "mais transdisciplinares em termos de objetivos (orientados para a sustentabilidade), métodos (das ciências naturais e sociais) e participantes (cientistas, profissionais, tomadores de decisão e partes interessadas de vários tipos)".

Osmond e Pelleri (2017, p. 40) complementam que a "ecologia urbana sustenta os desafios multigeracionais de manter e aprimorar a resiliência e a habitabilidade de nossos espaços urbanos e a saúde e bem-estar de seus habitantes". Debater e pesquisar sobre a Ecologia Urbana pode proporcionar uma compreensão dos ecossistemas urbanos e do seu funcionamento por meio de uma abordagem interdisciplinar.

Nesse sentido, as discussões sobre como a Ecologia Urbana pode atuar para promover a resiliência das cidades foram debatidas no Primeiro Congresso Mundial da Sociedade de Ecologia Urbana, realizado em Berlim no ano de 2013, e reuniu importantes pesquisadores da área a fim de estabelecer estratégias para as pesquisas futuras (McPhearson et al., 2016). A realização do evento em 2013 é relativamente recente, ressaltando a visibilidade que a Ecologia Urbana tem conquistado no cenário mundial.

Os debates e conferências ambientais sobre a Ecologia Urbana são importantes, pois as cidades estão em constante processo de crescimento e desenvolvimento, gerando pressões diárias nos ecossistemas naturais e construídos (Wu; Xiang e Zhao, 2014; Baumeister; Gerstenberg; Plieninger e Schraml, 2020). Em virtude disso, a Ecologia Urbana pode auxiliar na minimização desses impactos ambientais, por meio de uma visão integrada dos ecossistemas (naturais e construídos/urbanos) que vise a sustentabilidade urbana.

Nessa perspectiva, considerando os diversos problemas que ocasionaram o desequilíbrio ambiental da civilização humana na atualidade, pode-se destacar o padrão de crescimento insustentável das cidades dentre os principais. Esse padrão de crescimento foi consolidado durante e após a Revolução Industrial e caminha para gerar o colapso da sociedade humana (Wu, 2014). Para evitar que isso ocorra, estratégias de mitigação desses impactos precisam ser adotadas e colocadas em prática.

A ciência da ecologia proporciona a base científica para que se possa aprofundar os conhecimentos e estudos dentro das perspectivas da cidade como um tipo de ecossistema e, portanto, criar subsídios que possibilitam a análise do seu metabolismo, incluindo os fluxos de matéria e energia, visando compreender os impactos desencadeados pela 
expansão urbana (Terradas; Franquesa; Parés e Chaparro, 2011).

Dessa forma, para uma melhor compreensão do estudo das cidades, Adler e Tanner (2015) subdividem o ecossistema urbano em hábitats construídos, caracterizados pela estrutura urbana, contendo edifícios e construções em geral; os hábitats de resíduos, constituídos pelos resíduos humanos; os hábitats verdes e aquáticos, constituídos predominantemente por plantas e água, respectivamente.

Nessa perspectiva, a Ecologia Urbana considera que as cidades são ecossistemas artificiais/culturais, tendo em vista que, para que possam existir e garantir a manutenção da vida, são dependentes de necessidades biológicas e culturais básicas que permitem a esses espaços sua própria dinâmica de funcionamento e crescimento (Batitucci et al., 2019).

Considerando a cidade como um ecossistema urbano, é necessário compreender também que ocorrem diversos processos metabólicos derivados da espécie humana e de suas atividades dentro da cidade, que se caracterizam pela entrada de água, combustível, oxigênio, energia e alimentos, sendo uma parte destes armazenada e outra liberada, principalmente, na forma de resíduos (Oliveira; Leite; Prasad e Ribeiro, 2008).

Adler e Tanner (2015) definem o metabolismo urbano fazendo uma analogia ao metabolismo dos organismos vivos. De acordo com os autores, assim como os organismos vivos possuem vias metabólicas de entrada, processamento e saída de alimentos, água e materiais, as cidades também possuem essas respectivas vias metabólicas, que são constituídas por processos humanos e produtos sociais convertidos e/ou utilizados para construção e manutenção das áreas construídas. Ao final desse metabolismo artificial urbano, resíduos biológicos e não biológicos são gerados e exportados para o ambiente, podendo causar, em muitas situações, problemáticas ambientais pela grande quantidade de nutrientes e subprodutos que não são absorvidos em sua totalidade pelo ecossistema urbano.

Destaca-se também que os ecossistemas urbanos que compõe as cidades estão amplamente degradados pelas ações antrópicas, contudo, integrando esses ecossistemas artificiais, ainda resistem ecossistemas naturais oferecendo serviços ecossistêmicos importantes para a manutenção do bem-estar humano e para a conservação da biodiversidade (Wu, 2014). Por isso, necessitam de medidas de intervenção e mitigação dos impactos a elas gerados, a fim de garantir a manutenção dos serviços ecossistêmicos para as gerações atuais e futuras.

Nessa perspectiva, Barot et al. (2019) afirmam que as cidades são áreas experimentais que necessitam ser estudadas e, por fim, $\mathrm{Wu}$ (2014) complementa que as cidades se tornaram laboratórios a céu aberto, com o desenvolvimento de pesquisas que proporcionaram a compreensão entre as relações de homem e natureza a fim de obter um padrão de sustentabilidade, e que "as cidades futuras refletirão quem somos, o que valorizamos e quão bem podemos "refazer" nosso mundo" (Wu, 2014, p. 219).

\section{Abordagens e perspectivas da Ecologia Urbana sobre o prisma ecológico e interdisciplinar}

A construção das cidades objetiva garantir a manutenção de necessidades básicas da espécie humana, como a segurança e a reprodução da vida em sociedade (Lawrence, 2003). Embora apresentem estruturas totalmente diferentes e variáveis, todas as cidades sofrem influência direta de processos ambientais e socioeconômicos.

McPhearson et al. (2016) alertam para os desafios da Ecologia Urbana, destacando que as medidas adotadas como sustentáveis para algumas cidades podem não ser eficazes para outras, devido a diversidade de problemas socioambientais e econômicos decorrentes do estágio de urbanização. Isso demonstra o nível de complexidade de se trabalhar buscando a efetivação da sustentabilidade ambiental nos ecossistemas urbanos.

A inserção da Ecologia Urbana no planejamento urbano pode proporcionar diversos benefícios para a sociedade, como a garantia da prestação dos serviços ecossistêmicos e da infraestrutura verde (Osmond e Pelleri, 2017). Para tanto, faz-se necessário incorporar os conhecimentos advindos das pesquisas em Ecologia Urbana com diferentes perspectivas e abordagens.

Nesse sentido, Wu (2008) destaca que a Ecologia Urbana possui três vertentes gerais de conhecimento e abordagem que orientam as pesquisas e metodologias da área. De acordo com o autor, todas essas vertentes gerais variam em virtude do procedimento metodológico empregado e da formação do pesquisador:

1. Ecologia na Cidade: perspectiva relacionada a bioecologia, estudando como os sistemas biológicos são afetados dentro das cidades;

2. Ecologia das Cidades como estruturas socioeconômicas: perspectiva socioecológica que visa a compreensão da cidade por meio de uma base ecológica;

3. Ecologia das Cidades como ecossistemas: aborda os sistemas urbanos visando compreender a cidade como um ecossistema construído e impactado; considera o espaço urbano em sua amplitude integrado por questões ambientais e sociais; se caracteriza pela ecologia da paisagem, que considera a heterogeneidade das paisagens nas cidades para estudar o ambiente urbano.

A Ecologia Urbana nas Cidades se restringe ao estudo de organismos biológicos e suas interações dentro da cidade, possuindo uma visão limitada no que tange ao entendimento da cidade enquanto ecossistema construído que, mesmo degradado e modificado, ainda pode oferecer serviços ecossistêmicos a população. Diferentemente dessa visão de estudo, mas ainda na perspectiva clássica da Ecologia das Cidades, observa-se que o enfoque é remetido a abordagem sociológica, utilizando conceitos ecológicos para tentar entender o ambiente urbano, se revestindo assim de metodologias tradicionais que originaram a Ecologia Urbana na Escola de Chicago em 1920.

Com os avanços dos estudos e pesquisas na área, houve a elevação do conhecimento sobre organismos biológicos urbanos e dinâmicas socioeconômicas dentro das cidades, 
proporcionando a Ecologia Urbana adquirir um novo viés, este último, voltado para a compreensão mais ampla das estruturas urbanas enquanto ecossistemas construídos, complexos e dinâmicos, que interagem no espaço urbano ofertando serviços ecossistêmicos e bem-estar social. Essa abordagem também possibilitou a capacidade de compreensão ampliada dos impactos e modificações humanas sobre a paisagem natural, originando a cada momento novas paisagens construídas integradas aos habitats naturais que também podem ser fontes de serviços ecossistêmicos para as cidades.

No que tange as principais contribuições da Ecologia Urbana na vertente da Ecologia das Cidades como ecossistemas, o enfoque interdisciplinar ganha destaque, pois pretende correlacionar os sistemas naturais aos sistemas socioeconômicos. Dentre as três abordagens mencionadas, pode-se observar que a Ecologia nas Cidades possui uma base mais disciplinar e com uma perspectiva voltada para a compreensão do modo de vida dos organismos dentro das cidades (Wu et al., 2014).

Por outro lado, $\mathrm{Wu}$ (2008) complementa que a Ecologia das Cidades como ecossistemas, possui uma abordagem de pesquisa mais direcionada para o entendimento e contribuições dos serviços ecossistêmicos e da biodiversidade urbana para o bem-estar humano, envolvendo conhecimentos das ciências naturais e sociais, ou seja, o homem é visto como parte integrante do ecossistema urbano. Essa ideia sintetiza o objetivo da Ecologia Urbana nos dias atuais.

De acordo com McPhearson et al. (2016), para que uma pesquisa possa ser caracterizada como de Ecologia Urbana com abordagem interdisciplinar (Ecologia da Cidade como ecossistemas), é necessário que ela aborde cinco critérios básicos, a saber: 1. deve possuir enfoque nos sistemas, e promover interações socioambientais e de infraestrutura urbana; 2. ter uma base interdisciplinar, incorporando a política, tecnologia, saúde e governança; 3 . deve possuir uma equipe multidisciplinar interessada nos objetivos da área; 4. deve direcionar os estudos para interações espaço-temporais; 5. deve buscar a diversificação dos métodos de estudo para compreender de forma mais aprofundada a complexidade dos ecossistemas urbanos.

Contudo, embora fique notória as contribuições e a ampla abordagem das pesquisas de Ecologia das Cidades, destaca-se também a importância da Ecologia nas Cidades, já que a abordagem da bioecologia oferece o conhecimento biológico necessário para o estabelecimento de ações de planejamento urbano e conservação ambiental. Sendo assim, Wu (2014) ressalta que os estudos de Ecologia na Cidade têm crescido nas últimas décadas e permanecerão em ascensão, juntamente com os estudos de sustentabilidade, haja visto que o futuro da humanidade é dependente desse conhecimento.

A Ecologia Urbana, seja ela com abordagem na cidade ou da cidade, torna-se fundamental para o planejamento urbano sustentável no ambiente construído e para o gerenciamento adequado do meio ambiente. Considerando que as cidades são atualmente habitats de grande biodiversidade, incluindo espécies ameaçadas de extinção, e onde reside, predominantemente, a espécie humana (Osmond e Pelleri, 2017). Com a crescente urbanização, o maior desafio se volta não para redução dos processos de degradação ambiental, mas, principalmente, para a necessidade de projetar e planejar cidades mais sustentáveis, adaptativas e resilientes ( $\mathrm{Wu}$, 2008).

Para tanto, a Ecologia Urbana tem sido importante no desenvolvimento de pesquisas voltadas à diversidade biológica dentro das cidades, visando subsidiar a conservação dessas espécies em associação ao planejamento urbano sustentável (Negri; Negri; Angeoletto, 2019).

Nessa perspectiva, outros autores como Wu et al. (2014) sintetizam como ocorre as relações entre a Ecologia das Cidades e Ecologia nas Cidades e a relação destas na promoção da Sustentabilidade das Cidades, destacando as respectivas contribuições de cada uma dessas vertentes de estudo para as pesquisas de Ecologia Urbana. Os autores retratam que a promoção da sustentabilidade urbana depende intrinsecamente da interação entre os conhecimentos derivados da Ecologia na Cidade e Ecologia da Cidade, no qual pode gerar como produto final o bem-estar humano, por meio da conservação dos serviços ecossistêmicos e biodiversidade.

A Ecologia Urbana é imprescindível para promover a conservação ambiental dentro da cidade e potencializar os serviços ecossistêmicos. Ela possibilita o aporte teórico necessário para a construção de cidades sustentáveis que possam crescer economicamente, minimizando os impactos socioambientais.

\section{Conclusão}

Os estudos de Ecologia Urbana se expandiram em meio ao estabelecimento de uma crise ambiental civilizatória, e a abordagem da Ecologia da Cidade e Ecologia na Cidade proporcionaram a construção do conhecimento básico para a promoção de cidades mais sustentáveis, que priorizem a conservação dos ecossistemas naturais e os serviços ecossistêmicos por eles prestados. Contudo, após um século de criação na Escola de Chicago, no ano de 1920, a Ecologia Urbana trilhou um longo caminho de conquistas e de representatividade na sociedade científica, social e política, coexistindo com a insustentabilidade das cidades, buscando traçar estratégias de reversão desses cenários.

Atualmente, a Ecologia Urbana se reveste de abordagens metodológicas, socioecológicas e interdisciplinares relativamente recentes, que são essenciais para a conservação dos ecossistemas e da biodiversidade dentro das cidades. Contudo, em alguns países do planeta Terra, especialmente o Brasil, os estudos de Ecologia Urbana ainda se apresentam de forma incipiente, necessitando de maior representatividade em busca de atender as demandas das cidades por ecossistemas ecologicamente equilibrados.

\section{Referências}

Adler, F. R., \& Tanner, C. J. (2015). Ecossistemas urbanos: princípios ecológicos para o ambiente construído. São Paulo: Editora Oficina de Textos.

Angeoletto, F., Essy, C., Sanz, J. P. R., Silva, F. F., Albertin, R. M., \& Santos, J. W. M. C. (2015). Ecología Urbana: la Ciencia Interdisciplinaria del Planeta Ciudad. Desenvolvimento em Questão, 13(32), 6-20. doi: 10.21527/2237-6453.2015.32.6-20.

Barot, S., Abbadie, L., Auclerc, A., Barthélémy, C., Bérille, E., Billet, P., 
Clergeau, P., Consales, J. N., Cottin, M. D., David, A., Devigne, C., Dham, V., Dusza, Y., Gaillard, A., Gonzalez, E.; Hédont, M., Labarraque, D., Bastard, A. M. L., Morel, J. L., Petit-berghem, Y., Rémy, E., RochelleNewall, E., \& Veyrières, M. (2019). Urban ecology, stakeholders and the future of ecology. Science of the Total Environment, 667, 475-484. doi: 10.1016/j.scitotenv.2019.02.410

Batitucci, T. O., Cortines, E., Almeida, F. S., \& Almeida, A. A. (2019). Agriculture in urban ecosystems: a step to cities sustainability. Ambiente \& Sociedade, 22, 1-20. doi: 10.1590/1809-4422asoc0277r3vu19L4AO

Baumeister, C. F., Gerstenberg, T., Plieninger, \& T., \& Schraml, U. (2020). Exploring cultural ecosystem service hotspots: Linking multiple urban forest features with public participation mapping data. Urban Forestry \& Urban Greening, 48, 1-19. doi: 10.1016/j.ufug.2019.126561

Eufrasio, M. A. (2013). Estrutura Urbana e Ecologia Humana: A Escola Sociológica de Chicago (1915-1940). ( ${ }^{\circ}$ ed.). São Paulo: Editora 34.

Fellowes, M. (2019). We must learn to reduce our demands on the ecosphere, but I'm not sure that we're smart enough to do that.Terr@Plural, 13(3), 26-29. doi: 10.5212/TerraPlural.v.13i3.0002

Grimm, N. B., Faeth S. H., Golubiewski, N. E., Redman, C. L., Wu, J., Bai, X., \& Briggs, J. M. (2008). Global change and the ecology of cities. Science, 319, 756-760. doi: 10.1126/science.1150195.

Haeckel, E. Generelle Morphologie der Organismen, Bd. 2:Allgemeine Entwickelungsgeschichte der Organismen. (1866). Reimer, Berlin.

Lawrence, R. J. (2003). Human ecology and its applications. Landscape and Urban Planning, 45, 31-41. doi: 10.1016/S0169-2046(02)00235-9

Leveau, L. M. (2020). Urban ecology in Latin America should be more interdisciplinary. Terr@ Plural, (14), 1-3. doi: 10.5212/TerraPlural.v.14.2014176.002

McPhearson, T., Pickett, S. T. A., Grimm, N. B., Niemelã, J., Alberti, M., Elmqvist, T., Weber, C., Haase, D., Breuste, J., \& Qureshi, S. (2016). Advancing Urban Ecology toward a Science of Cities. BioScience, 66(3), 198-212. doi: 10.1093/biosci/biw002

Negri, I. C. O., Negri, S. S., \& Angeoletto, F. (2019). As cidades médias brasileiras precisam ser mais amigáveis aos morcegos, principalmente nos hotspots de biodiversidade. Terr@ Plural, 13(3), 446-460. doi: https://doi.org/10.5212/TerraPlural.v.13i3.0029

Niemelä, J. (1999). Ecology and urban planning. Biodiversity and Conservation, 8, 119-131. doi: 10.1023/A:1008817325994

Niemelä, J. (2014). Ecology of urban green spaces: The way forward in answering major research questions. Landscape and Urban Planning, 125, 298-303. doi: 10.1016/j.landurbplan.2013.07.014

Nucci, J. C. (2007). Origem e desenvolvimento da Ecologia e da Ecologia da Paisagem. Revista Eletrônica Geografar, 2(1), 77-99. doi: 10.5380/geografar.v2i1.7722

Oliveira, S. A., Leite, V. D., Prasad, S., \& Ribeiro, M. D. (2008). Serviços de Limpeza Urbana: aspectos sociais, econômicos e ambientais. Gaia Scientia, 2(1), 41-49.

Osmond, P., \& Pelleri, N. (2017). Urban Ecology as an Interdisciplinary Area. Encyclopedia of Sustainable Technologies, 2, 31-42. doi: 10.1016/B9780-12-409548-9.10173-3

Park, A., Burgess, E., \& Mckenzie, R. (1925). The City. University of Chicago Press: Chicago, 1925.

Pickett, S. T. A. (2020). It is important that medium-sized places have the resources to improve the ecological quality of their growth.Terr@Plural, (14), 1-4. doi: 10.5212/TerraPlural.v.14.2014178.003

PNUMA. Programa das Nações Unidas para o Meio Ambiente. (2020).

Rodrigues, E., \& Victor, R. A. B. M. Os serviços dos ecossistemas e sua importância para o bem-estar humano no cinturão verde da cidade de São Paulo. ( $1^{\mathrm{a}}$ ed. São Paulo: Instituto Florestal, 2014.

Rumble, H., Angeoletto, F., Connop. S., Goddard, M. A., \& Nash, C. (2019). Understanding and Applying Ecological Principles in Cities. Planning Cities with Nature, 2017-234. doi: 10.1007/978-3-030-01866-5

Silva, I. P., \& Bomfim, L. S. V. (2019). O Télos da Ecologia Humana no Brasil e sua interface com as populações tradicionais. Acta Brasiliensis, 3(1), 3539. doi: $10.22571 / 2526-4338151$

Steiner, F. (2008). Human Ecology: Overview. Encyclopedia of Ecology, 18981906. doi: 10.1016/B978-008045405-4.00626-1
Terradas, J., Franquesa, T., Parés, M., \& Chaparro, L. (2011). Ecología Urbana: Considerar una ciudad como un ecosistema ayuda a entender su funcionamiento y resulta esencial para diseñar estrategias de futuro y vigilar su desarrollo. Investigación y Ciencia, 52-60.

Tonyaloglu, E. E. (2020). Spatiotemporal dynamics of urban ecosystem services in Turkey: The case of Bornova, Izmir. Urban Forestry \& Urban Greening, 49, 1-10. doi: 10.1016/j.ufug.2020.126631

Wu, J. (2008). Making the Case for Landscape Ecology: An Effective Approach to Urban Sustainability. Landscape Journal, 27, 41-50.

Wu, J. (2014). Urban ecology and sustainability: The state-of-the-science and future directions. Landscape and Urban Planning, 125, 209-221. doi: 10.1016/j.landurbplan.2014.01.018

Wu, J., Xiang, W. N., \& Zhao, J. (2014). Urban ecology in China: Historical developments and future directions. Landscape and Urban Planning, 125, 222-233. doi: 10.1016/j.landurbplan.2014.02.010

Licença: Creative Commons CC BY NC 4.0 